\title{
Aportes del modelo de Yrjö Engeström al desarrollo teórico de la docencia universitaria
}

\author{
Contributions of Yrjö Engeström's Model \\ to the Theoretical Development of University Teaching
}

\author{
Flora Eugenia Salas-Madriz \\ Universidad de Costa Rica \\ Vicerrectoría de Docencia \\ San José, Costa Rica \\ fsalasmadriz@gmail.com \\ ORCID: orcid.org/0000-0003-2163-0370
}

\section{Recibido: 11 julio 2014 Aceptado: 22 octubre 2015 Corregido: 7 marzo 2016}

\begin{abstract}
Resumen: Este artículo resume los aportes epistemológicos y teóricos de los capítulos $I V$ y $V$ de la tesis doctoral titulada La integración de las TIC en la docencia en la UCR: Estudio desde un enfoque sistémicocomplejo del personal docente que se certificó en el curso Educar para el Futuro, versión universitaria en el período 2003-2006, que se defendió en el 2013. Para ese efecto, se explica el modelo de un sistema de enseñanza y aprendizaje, desarrollado por Yrjö Engeström (Universidad de Helsinki), que integra principios de la teoría de sistemas (TS), la teoría de la complejidad (TC), la teoría sociocultural (TS) y la teoría de la actividad (TA), y se revisan sus aportes a la descripción, explicación y comprensión de la docencia universitaria, entendida como una actividad humana particular, que ocurre dentro de un sistema de enseñanza y aprendizaje, regido por estructuras, funciones y dinámicas relacionales que la determinan y la hacen posible en contextos, a su vez, complejos. El principal aporte de este esfuerzo teórico es que, si bien se asume la docencia como un fenómeno social de altísima complejidad, no por ello es inaccesible a la investigación científica; por el contrario, a través del modelo de Engeström se abren nuevas posibilidades para el desarrollo de tecnologías -entendidas como conocimiento científico aplicado-, para solventar problemas fundamentales de la docencia en la educación superior contemporánea, donde la necesidad de definir perfiles y diseñar estrategias para la formación pedagógica para el personal académico son desafíos para la gestión del cambio y la innovación en esas instituciones. Otro elemento relevante del modelo de Engeström es que supera visiones tradicionalistas, reduccionistas y mecanicistas de la docencia universitaria, lo que favorece soluciones realistas, efectivas $y$ coherentes con las demandas educativas emergentes y los nuevos perfiles del profesorado universitario impulsados por el ingreso en la sociedad de la información y el conocimiento (SIC).
\end{abstract}

Palabras clave: Yrjö Engeström, teoría de la actividad, teoría de sistemas, sistemas de enseñanza y aprendizaje, docencia universitaria, cambio e innovación en educación superior.

Abstract: This article shows the epistemological and conceptual contributions of chapters $I V$ and $V$ of the doctoral thesis titled The Integration of ICT's in Teaching at UCR: A study from a complex-systemic approach of the faculty who got certified in the course Teach to the Future, university version in the timeframe 2003-2006, which was disserted in 2013. For this reason, the model for a system of teaching and learning developed by Yrjö Engeström (University of Helsinki) is discussed. This model integrates

1 Licenciada en Filosofía (UCR) y Psicología (Universidad Centroamericana de Ciencias Sociales (UCACIS); tiene una Maestría en Administración Universitaria (UCR) y es Doctora en Educación (UCR). Es profesora Catedrática de la UCR (2009) y desde 1994 ha trabajado como docente e investigadora en la Sede del Atlántico, la Escuela de Estudios Generales y la Escuela de Administración Educativa de la Universidad de Costa Rica. De junio de 2012 a agosto de 2013, se desempeñó como asesora académica en la Vicerrectoría de Docencia de la Universidad de Costa Rica. A partir del 01 de setiembre de 2013, ocupa el cargo de directora de Gestión de Procesos, Políticas y Proyectos en la Vicerrectoría de Docencia de la Universidad de Costa Rica. 
the principles of Systems Theory, Complexity Theory, the Sociocultural Paradigm and the Activity Theory (AT). The contributions of this model to the description, explanation, and understanding of university teaching, conceived as a "particular human activity" occurring within a "system of teaching and learning", governed by structures, functions and relational dynamics are also reviewed. As well as how they define and make it possible to determine internal and external contexts which are, in turn, complex. Assuming teaching as a phenomenon of high complexity, but approachable to scientific research through Engeström's model, opens limitless possibilities to the development of technologies-understood as applied scientific knowledge- to solve fundamental problems of teaching in contemporary higher education. Today, the need to define profiles and create strategies for the academic professors of universities has become one of the biggest challenges in the management of change and innovation in higher education. Hence, another relevant aspect of theoretical models is the ability to overcome the traditional, reductionist and mechanistic teaching practices in higher education in order to find realistic, effective and consistent solutions for emerging educational demands and new profiles of the university faculty, driving entry into a new phase of socio-historical development, known as the Information and Knowledge Society.

Keywords: Yrjö Engeström, Activity Theory, Systems Theory, Sociocultural Theory, systems of teaching and learning, university teaching, change and innovation in higher education.

\section{Introducción}

A lo largo del siglo XX, la investigación educativa pasó del enfoque empírico-analítico hacia el sistémico-complejo, dejando entre ellos una cantidad importante de hallazgos de investigación de corte cualitativo, donde predominaron el enfoque fenomenológico, etnográfico, hermenéutico y el sociocrítico. Gracias a los aportes de la investigación cualitativa, conforme la complejidad del fenómeno educativo se describió, explicó y comprendió con mayor precisión en su estructura, componentes, relaciones y funciones propias, resultó evidente que no era posible dar cuenta de él a partir de enfoques epistemológicos y teóricos que proponían una perspectiva reduccionista, fragmentada y parcial de los hechos sociales (Delgado y Gutiérrez, 1998). De esta forma, responder a la pregunta que da a la educación su objeto de estudio: ¿Cómo enseñar al ser humano ciertos conocimientos, habilidades y destrezas de manera intencionada?, implicó explorar posiciones teóricas, cuyas epistemologías subyacentes tuviesen como posibilidad una visión global del hecho educativo, capaz de dar cuenta de sus principales componentes y relaciones, así como del contexto interno y externo que subyace a toda práctica de la enseñanza en los ámbitos formal y no formal.

El enfoque sistémico-complejo de la educación, posible a partir de los aportes de la teoría de sistemas (Luhmann), la teoría de la complejidad (Morin) y la teoría de la actividad (Alekséi Leóntiev), que desarrolló Yrjö Engeström, permitió la creación de un modelo teóricoconceptual capaz de describir la estructura, componentes y relaciones de la actividad humana en sistemas de enseñanza y aprendizaje, que presupone y se explica a partir de la capacidad de enseñar y aprender de nuestra especie. Desde esta perspectiva, existen supuestos en educación, entendida como un sistema de enseñanza y aprendizaje intencionado, articulado y contextualizado, que hacen posible la construcción de un marco explicativo para el fenómeno educativo en todas sus dimensiones (Salas, 2013); entre ellos, destacan los siguientes:

El ser humano:

- es un ser vivo social determinado por la herencia biológica y cultural;

- es inteligente, ya que posee un sistema nervioso altamente desarrollado y complejo que le faculta para realizar procesos de pensamiento consciente y racional; 
- es capaz de acceder al lenguaje y de comunicarse consigo mismo y con otros seres humanos, así como de articular procesos cognitivos y de pensamiento con base en herramientas simbólicas, a través de las cuales se transforma a sí mismo;

- tiene la posibilidad de construir medios y herramientas materiales para satisfacer necesidades de distinta índole, a partir de las cuales transforma su entorno social y natural;

- es capaz de una vida psíquica que le permite comprender y experimentar la diferencia yo/mundo y, en consecuencia, el desarrollo de la capacidad de autopoiesis, autoconsciencia y autorregulación;

- tiene la capacidad de aprender desde que nace hasta que muere por cuenta propia, por acumulación de experiencia y por la intermediación de otros seres humanos;

- puede y tiene la capacidad de aprender "ciertos conocimientos, habilidades y destrezas", si ello es necesario para su supervivencia física, psíquica y social, tiene interés o lo desea a título personal; $y$,

- tiene la capacidad de enseñar a otros seres humanos lo que sabe, si ello es necesario, tiene interés o lo desea a título personal.

Como consecuencia de lo anterior:

- La enseñanza y el aprendizaje son funciones vitales, porque constituyen actividades indispensables para la supervivencia de la especie humana y, por ende, de los individuos que forman parte de ella.

- Toda actividad humana es una "actividad mediada" por herramientas simbólicas y materiales, que dan acceso a objetos del "mundo de la vida" y al logro de objetivos (García y García, 1996; Habermas, 1988; Luhmann, 1998).

A partir de la descripción de los supuestos básicos de la educación desde una perspectiva bio-psico-social, la epistemología del enfoque sistémico-complejo sobre el fenómeno educativo que propone Engeström se fundamenta en el materialismo histórico-dialéctico, la TA y el paradigma sociocultural de Vigotsky; situación que ofrece posibilidades inéditas en la historia de esta disciplina para comprender el fenómeno educativo en su complejidad y dimensiones primordiales. El materialismo histórico rompe con la tradición epistemológica greco-occidental, que propone que el conocimiento ocurre de manera deshistorizada, más allá de la biografía del sujeto cognoscente y del grado de desarrollo de conocimiento alcanzado sobre el problema u objeto de estudio. La TA explica el "carácter mediado de la acción y, [en consecuencia], para esclarecer cómo conoce y aprende el ser humano es preciso describir y explicar, además del impacto de la carga genética, la forma como se configuran y articulan la psique, la biografía y el contexto sociocultural" (Salas, 2013, p. 104). El paradigma sociocultural permite una aproximación al hecho educativo donde el ser humano es concebido a partir de los supuestos descritos anteriormente, y se asume que la inteligencia es un atributo biológico que se desarrolla para adquirir conocimientos social y personalmente relevantes, a partir del 
contacto con el saber social al que se accede a lo largo de la vida, gracias al contacto con la cultura y otros seres humanos.

En el enfoque sistémico-complejo, "la actividad humana es parte de un sistema que involucra lo biológico, lo psicológico, lo cognitivo, lo socio-histórico y lo cultural” (Salas, 2013, p. 104); de ahí su potencial para hacer un abordaje teórico-conceptual innovador de la docencia, así como de sus implicaciones en los distintos niveles y componentes de un sistema de enseñanza y aprendizaje.

\section{Postulados de la teoría de la actividad (TA)}

La TA propone que la acción humana ocurre en un sistema social y que ella no se da en el "vacío", ni al margen de la propia conciencia y la realidad sociohistórica y, por consiguiente, es siempre "actividad mediada". Al respecto, el psicoanálisis (Freud, 1981) demostró en los niveles teórico y práctico, que ante procesos psíquicos patológicos las motivaciones inconscientes también operan como detonantes de la actividad humana. Cuando esas motivaciones se explican a través del proceso terapéutico, resulta clara la línea de acción del sujeto y se revelan los mecanismos simbólicos utilizados para el logro de resultados particulares, consistentes con la patología que sufre la persona. La actividad consciente e inconsciente del ser humano se rige por los mismos principios, aunque sus resultados difieran sustancialmente en términos de logros y objetivos. Este es otro elemento relevante de esta teoría, pues permite explicar y comprender procesos de actividad humana conscientes e inconscientes.

Para la ejecución de cualquier actividad, el ser humano emplea determinados procedimientos que configuran sistemas de acción y operación que dependen de él mismo, las características del objeto u objetivo, los medios disponibles, los modos de proceder y de las condiciones del entorno interno y externo. Los medios son los instrumentos materiales, informativos, lingüísticos (simbólicos) y psicológicos que posee la persona, que están a su disposición para la transformación de objetos concretos y la consecución de objetivos. Las condiciones del entorno son el conjunto de situaciones de naturaleza ambiental, cultural y social donde se efectúa la actividad. El entorno interno lo constituyen las motivaciones psico-afectivas y racionales del sujeto. Finalmente, los productos son los resultados de la actividad.

Los objetos u objetivos son el principio del sistema de la actividad humana, porque relacionan sus componentes entre ellos y le dan una dirección determinada hacia un resultado final, que se asume posible. Debido a que la actividad humana es social, tiene como base una comunidad regida por ciertas reglas y formas establecidas de acuerdo con la división del trabajo necesaria para producir y reproducir el sistema social. Esto significa que las acciones humanas están prescritas por pautas de comportamiento social históricamente determinadas, que le dan acceso a herramientas materiales y simbólicas producto del acervo cultural y del grado de desarrollo de las fuerzas productivas.

En las figuras 1 y 2, se representan los componentes básicos de la actividad humana, donde un supuesto clave es que el sujeto utiliza herramientas materiales y simbólicas para aproximarse a los objetos y la realidad externa para lograr metas u objetivos. Debido a que la actividad humana es en principio consciente, dado un margen de libertad determinado, para 
la consecución de sus objetivos es posible aceptar o no las reglas de la comunidad, plegándose a la división social del trabajo que exista en ella. La suma de esos factores hace posible la actividad humana y la obtención de resultados motivados interna y externamente.

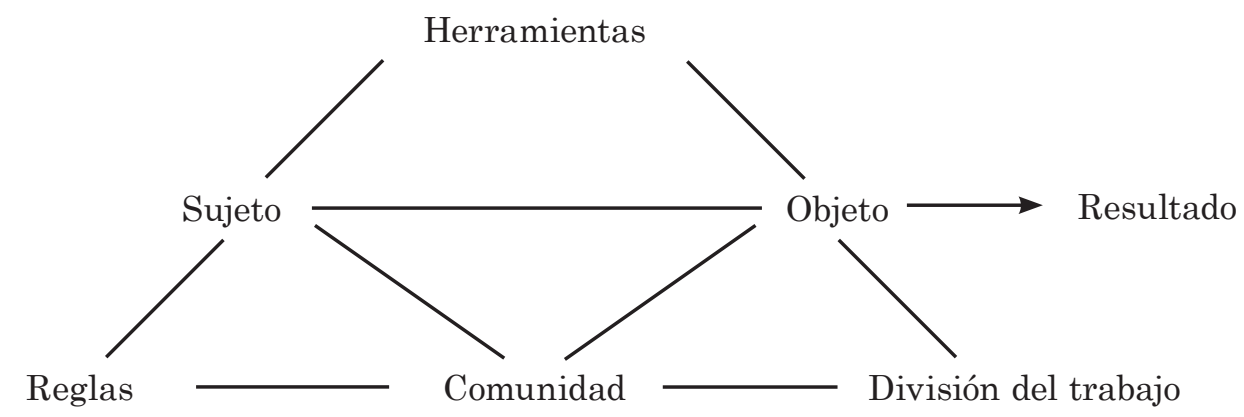

Figura 1. Estructura básica de la actividad humana (Vizcarro y León, 1998, p. 203).

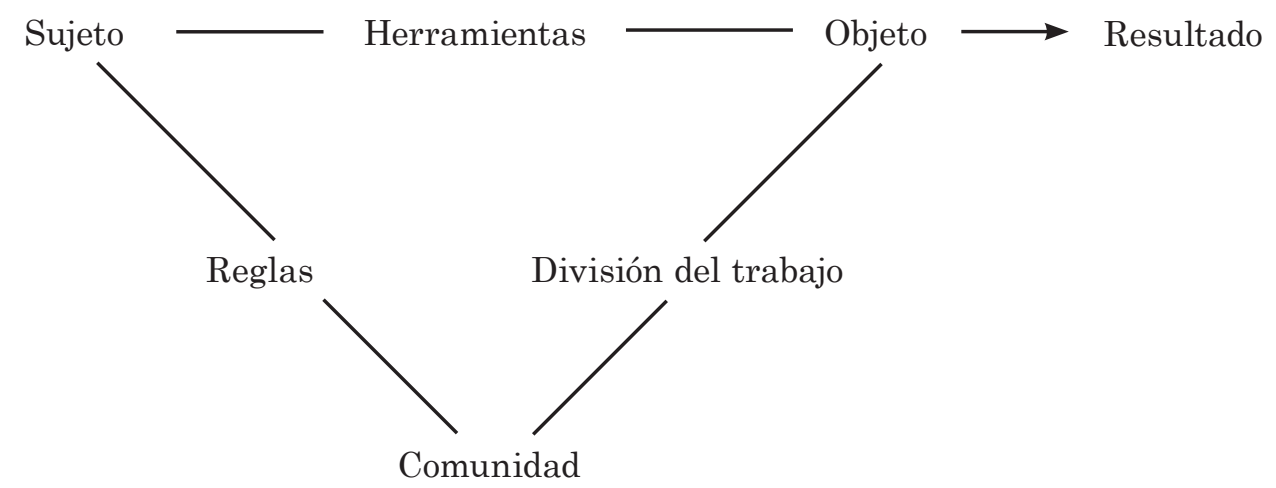

Figura 2. Elementos de la actividad humana (Vizcarro y León, 1998, p. 203).

Por sus características, la actividad humana tiene tres niveles jerárquicos: actividad, acción y operación, que pueden ser individuales o grupales (cooperativos) y corresponden a motivaciones, metas y condiciones particulares. En una actividad "(el elemento global) puede conseguirse mediante diversas acciones y la misma acción puede ser empleada en actividades diferentes" (Vizcarro y León, 1998, p. 204); desde esta perspectiva, las operaciones tienen las mismas características de la actividad. Los niveles jerárquicos de la TA son:

1. "El nivel intencional se orienta hacia los motivos: necesidades, deseos, valores. Es el nivel de orientación global el que da significado a los procesos humanos. 
2. El nivel procedimental está orientado hacia metas conscientes, específicas, dentro del marco de los motivos. Es el nivel de los procesos de organización, planificación y solución de problemas para lograr una meta final o metas intermedias.

3. El nivel operacional está orientado a las condiciones prácticas de la acción. Es el nivel de las rutinas prácticas que son un requisito previo a las acciones conscientes, con un fin determinado en el nivel funcional." (Vizcarro y León, 1998, p. 204)

La TA es un marco de referencia teórico-conceptual dinámico, en el sentido de que "las acciones humanas pueden desplazarse de un nivel a otro como resultado de frustraciones en relación con sus objetos [u objetivos]. Las 'frustraciones' o contradicciones pueden ser resultado de factores externos cambiantes" (Vizcarro y León, 1998, p. 205). Las contradicciones, generalmente, son consecuencia "de la interconexión del conjunto de actividades con situaciones de la vida real. Cada miembro de la comunidad participa en múltiples marcos de actividad, que incluyen comunidades múltiples, y los desarrollos de un marco de actividad pueden influir y conducir a contradicciones en otros marcos" (Vizcarro y León, 1998, p. 205).

En la TA la frustración y las contradicciones pueden actuar como freno o estímulo para el desarrollo de la actividad humana en los niveles intencional, funcional y operacional. Por su fundamento epistemológico y teórico, como se expuso, este enfoque se puede utilizar para el estudio de la actividad individual y grupal, y a partir de los supuestos del modelo de Engeström es posible construir una estructura general de la actividad humana, que integra sus componentes esenciales, con base en los cuales se explica cómo ella es posible en un contexto sociohistórico dado, como se muestra en la figura 3. Si se ajusta el modelo a un sistema de enseñanza y aprendizaje, para el caso de la docencia, quedaría como se muestra en la figura 4.

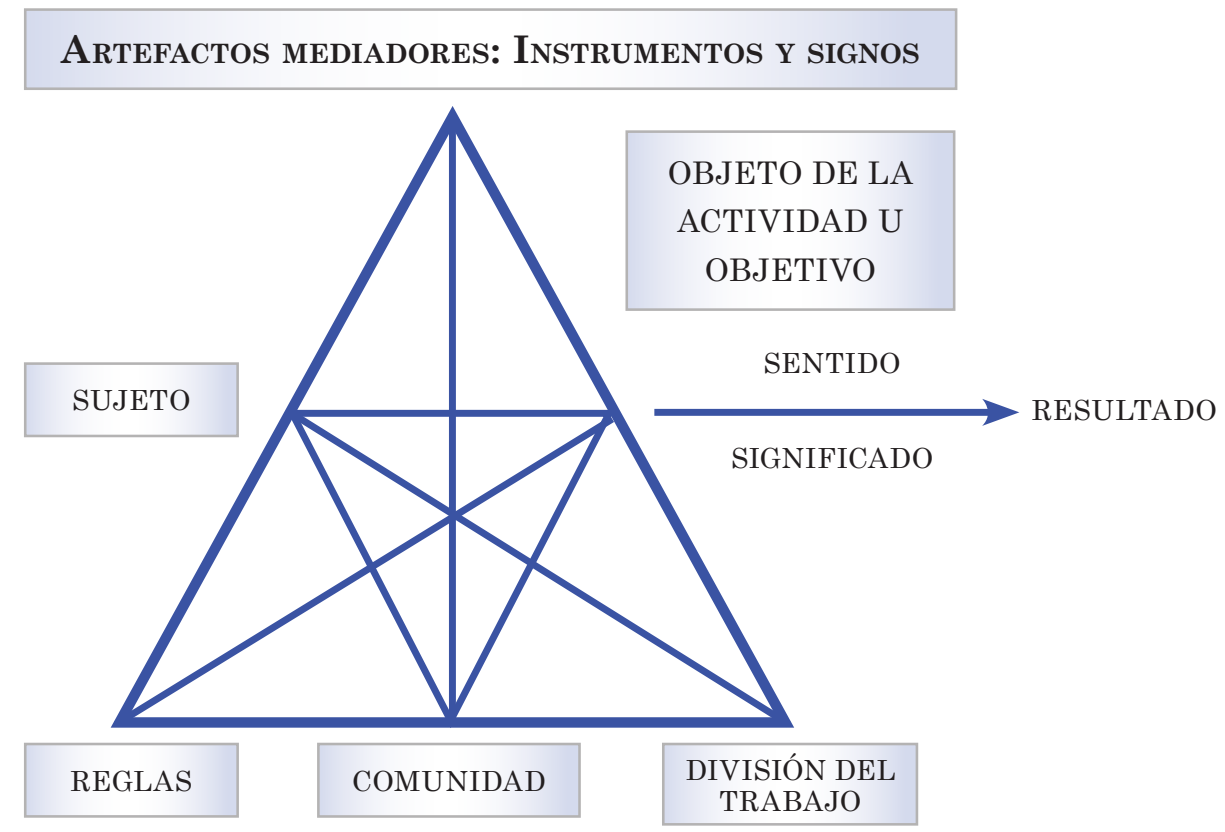

Figura 3. Elementos de la actividad humana. Elaboración propia con base en Knight (2006). 


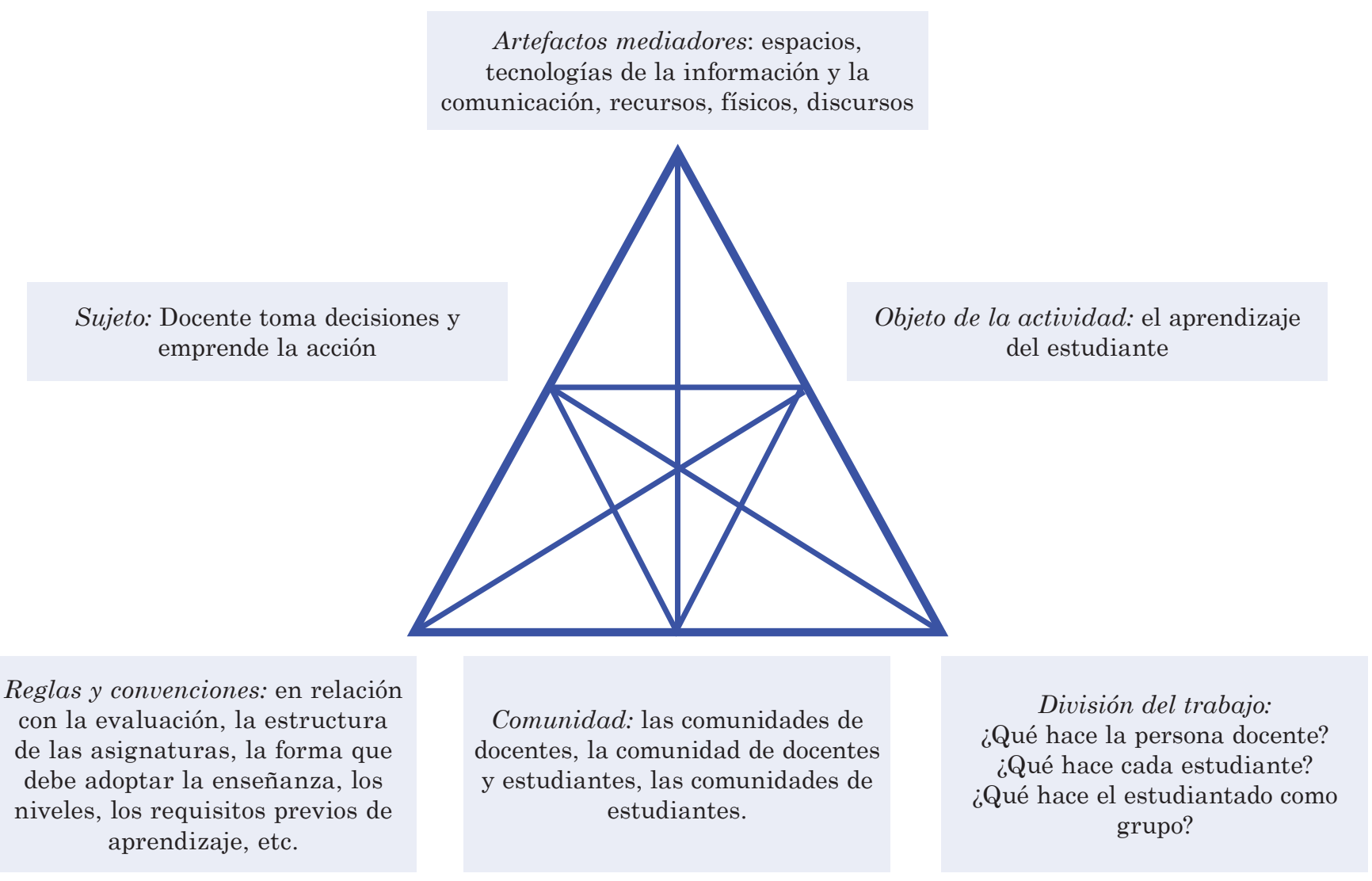

Figura 4. Actividad docente en un sistema de enseñanza y aprendizaje.

Elaboración propia con base en Knight (2006, p. 40).

\section{Aplicaciones de la TA al análisis sistémico-complejo de la docencia}

El profesorado lo constituyen individuos con conciencia de sí, características particulares y relaciones con otros seres humanos, que están inmersos en un contexto sociocultural. Su entorno interno lo integran el estudiantado, la comunidad docente y la institución educativa. En tanto personas que "enseñan", tienen como función la administración y ejecución de la docencia; es decir, la planificación y práctica del proceso de enseñanza, con el objetivo de que el estudiantado adquiera saberes previstos.

La práctica docente o actividad del profesorado tiene como particularidad de que, debido a ella, "se transforma no un objeto material inanimado, sino un ser humano, una persona que se modifica a sí misma con la ayuda de otras personas más capaces, especialmente con la guía, orientación y mediación del profesor" (Vidal, 2007, p. 9). Esto significa que el objeto de la actividad del personal docente es la dirección y control del aprendizaje del estudiantado a su cargo. Por ello, debe tener un concepto de quién y cómo es la persona aprendiz y una teoría -que puede ser científica o intuitiva- sobre cómo aprende el ser humano, pues sobre esa base establece los criterios fundamentales para orientar la acción como "enseñante", que constituye su función específica dentro del sistema de enseñanza y aprendizaje. 
Los motivos del profesorado son diversos, pero siempre están vinculados con sus valores, visión de la enseñanza y su rol asignado, así como con los valores y la "visión" de la enseñanza de la institución, el enfoque del currículo, la cultura académica, y los incentivos y acciones administrativas que favorecen o limitan la calidad y efectividad de la actividad docente. Los objetivos de la enseñanza, en principio, deben estar alineados y ser coherentes con los objetivos de aprendizaje, en respuesta a las demandas educativas que satisface la institución; son importantes porque "llegan a constituir verdaderamente el elemento rector del proceso de enseñanza y aprendizaje, cuando tanto los alumnos como los profesores los asumen conscientemente como suyos" (Vidal, 2007, p. 4). Con base en lo anterior, de acuerdo con el modelo de Engeström, la actividad del profesorado comprende:

1. El conocimiento y preparación de los contenidos de la materia que imparte, lo que presuponen una teoría de la educación y una práctica pedagógica.

2. El conocimiento psicopedagógico y de las características del alumnado que atiende.

3. La conciencia de los objetivos a lograr y de las condiciones ambientales y sociales que se pueden dar a lo largo del proceso de enseñanza.

4. La selección de procedimientos, tareas y medios más adecuados para una enseñanza efectiva (Vidal, 2007).

Los procedimientos de la enseñanza son "los métodos, técnicas y estrategias pedagógicas que planifica, organiza e introduce el profesor en el proceso para propiciar el aprendizaje de sus alumnos, regularlo y corregirlo" (Vidal, 2007, p. 5); debido a esto, se debe garantizar que correspondan a los contenidos curriculares previstos, así como a las características y requerimientos del alumnado. Los medios pedagógicos son "los recursos materiales, informativos, lingüísticos y psicológicos que emplea el profesor para facilitar una comunicación educativa eficaz con sus alumnos y, con ello, el proceso de interiorización de los contenidos de un plano social a un plano individual" (Vidal, 2007, p. 5).

Las condiciones de la enseñanza se relacionan directamente con el espacio físico y social de la institución; no obstante, el personal docente tiene injerencia sobre estos aspectos, en particular, en el nivel microsocial del aula, donde su responsabilidad es crear un ambiente agradable y cómodo para que el estudiantado lleve a cabo su aprendizaje con la tranquilidad y estabilidad emocional e intelectual que ello requiere. Por su parte, los productos del proceso de enseñanza y aprendizaje son, además de los conocimientos, habilidades y destrezas planeadas, "las transformaciones logradas tanto en la personalidad del estudiante y en la actividad del profesor, como en el proceso mismo" (Vidal, 2007, p. 5).

La actividad del profesorado se compone de cuatro momentos fundamentales: orientación, ejecución, control y corrección, y es la resultante del desarrollo de una base de orientación para la acción de la enseñanza, que involucra las acciones que llevan a cabo docentes y estudiantes. La enseñanza incluye la planificación y organización global del trabajo pedagógico, que se exterioriza en el enfoque pedagógico, el currículo y los programas de curso, y son tareas fundamentales en la actividad del profesorado. La planificación 
consiste en la búsqueda de las condiciones necesarias para la enseñanza. La organización comprende tomar en cuenta el espacio, el tiempo, el diseño de las tareas o actividades de enseñanza y la evaluación, así como la dirección y el control de los procesos que ello implica (Vidal, 2007).

Planear significa que el profesorado debe "establecer qué debe hacerse durante el proceso de enseñanza y aprendizaje, cómo debe desarrollarse, mediante qué acciones concretas y sus responsables, atendiendo a las metas y a la misión de la institución educativa" (Vidal, 2007, p. 6). La ejecución del personal docente es, entonces, la realización de lo planificado y organizado de manera flexible, atendiendo las vicisitudes que se presenten a lo largo de la sesión de clase y del curso como un todo. La función principal del profesorado es, desde esta perspectiva, la regulación o control sistemático del proceso de enseñanza y aprendizaje en su totalidad: respecto del aprendizaje del estudiantado y de la propia actividad de enseñanza; es decir, es un proceso autopoiético y requiere de la reflexión y el análisis permanente.

La actuación del personal docente está sometida a las contingencias propias de los sistemas abiertos, razón por la cual la organización prevista del proceso de enseñanza y aprendizaje, por detallada que sea, queda sujeta a las tendencias a la desorganización condicionadas por factores externos que escapan al control del profesorado; por esta razón, las contingencias y contradicciones ponen en juego las "motivaciones" y "expectativas" del personal docente, en la medida en que apelan a factores psicológicos (emociones y sentimientos) que favorecen o limitan la persistencia en el propósito; en consecuencia, la actitud y motivaciones de naturaleza intrínseca son esenciales en el éxito o fracaso de la acción docente. En este nivel, "la comunicación educativa juega un papel preponderante, porque permite crear una red de relaciones interpersonales afectivas que facilitan el compromiso de los participantes con el logro de lo planificado" (Vidal, 2007, p. 7).

El papel que desempeña el profesorado es fundamental, porque dirige y lidera el proceso de enseñanza y aprendizaje; también, de las decisiones que tome y las actitudes que muestre dependerán, en gran medida, la calidad del aprendizaje y el tipo de formación que reciba el estudiantado. Esto permite concluir que la actitud, la actuación y la capacidad del profesorado para motivar a sus estudiantes influyen en el éxito y fracaso académico, y que ello queda condicionado por el entorno interno y externo del sistema de enseñanza y aprendizaje. Asimismo, que el profesorado tenga autonomía "relativa" dentro del sistema de enseñanza y aprendizaje para orientar su práctica docente evidencia que está sujeto a las reglas y convenciones de la institución, a la comunidad académico-estudiantil y a la división del trabajo docente; situaciones que se suman a las condiciones generales de trabajo, al enfoque curricular y pedagógico predominante, y al acceso a los artefactos mediadores.

De acuerdo con lo anterior, la investigación señala que, en general, para relacionarse adecuadamente con el estudiantado el personal docente debe:

5. Tener en cuenta las particularidades individuales, la experiencia personal, las necesidades y la actividad del alumno.

6. Trabajar con el grupo como un todo, sin abandonar el enfoque personal en el tratamiento de los estudiantes. 
7. No ser estereotipado ni en la conducta, ni en los juicios.

8. No ser selectivo en los contactos, ni subjetivo en las valoraciones.

9. No ser agresivo en las relaciones.

10. Estimular la participación activa de los estudiantes en la manifestación de criterios, tanto de temas docentes como de otra índole.

11. Preocuparse por los problemas y dificultades de los estudiantes, tanto en el área docente como en lo personal y familiar.

12. Ser sensible y tener tacto en el trato.

13. Destacar más los logros que las insuficiencias.

14. Encaminar su actividad a la formación integral de la personalidad del alumno. (Vidal, 2007, p. 7)

La actuación del profesorado también está asociada al control del proceso de enseñanza y aprendizaje, que radica en la constatación del aprendizaje del alumnado, respecto de los objetivos planteados inicialmente. En ese proceso, se verifica el grado de efectividad del profesor o profesora. Una baja promoción, una alta deserción y el bajo rendimiento académico son indicadores de una mala ejecución docente; de ahí que los resultados de la evaluación de los aprendizajes, además de determinar el desempeño del alumnado, sirven como indicadores del desempeño docente. El control cumple con las siguientes funciones: indica la efectividad del trabajo con base en la planificación, la organización y la regulación; evidencia aspectos que se deben mejorar y revela el grado de eficacia de las actuaciones frente a contingencias.

Hay dos tipos de control en el proceso de enseñanza y aprendizaje: interno y externo. El primero es el que lleva a cabo el profesorado sobre su propio proceso de enseñanza, que se refleja en dos niveles: el aprendizaje del alumnado y la propia conciencia, y la autorreflexión sobre la actividad docente. El segundo lo ejercen los agentes autorizados oficialmente para evaluar y certificar la calidad y eficacia del proceso global de enseñanza y aprendizaje en las instituciones educativas (Vidal, 2007). Las diferencias entre los resultados obtenidos y los esperados son útiles para identificar el nivel de logro de la enseñanza y el aprendizaje, así como las áreas que requieren mejora en el personal docente, el alumnado y la institución.

Debido a la acelerada obsolescencia del conocimiento, a los profundos y constantes cambios en las demandas educativas y a la diversidad de estudiantes que acuden a las instituciones educativas, la formación continuada del profesorado es indispensable; máxime si se considera el grado de influencia que tiene su actuación sobre la calidad, el éxito y las características de la enseñanza.

\section{El modelo de Engeström: La enseñanza como componente de un sistema de enseñanza y aprendizaje en educación superior}

Entendemos la enseñanza como un sistema de trabajo complejo constituido por "una red de comunicaciones, actividades, creencias y otras cuestiones relacionadas" (Knight, 2006, p. 15), que ejecutan personas particulares para desarrollar procesos de enseñanza y aprendizaje 
intencionados e institucionalizados. Sobre ese sistema de trabajo inciden otros subsistemas, a la vez complejos, como la estructura y la cultura de la organización educativa, la disciplina que se enseña, el enfoque curricular, la visión y funciones de la enseñanza, las características del estudiantado, la estructura, funciones y dinámica administrativa, y el contexto sociocultural en el que está inmersa la institución educativa.

Al hablar de personas que forman parte de una organización educativa universitaria -docentes, estudiantes, personal docente-administrativo, personal de apoyo, comunidades académicas- y de los subsistemas que la conforman -la institución, las unidades académicas, las salas de clase o aulas-, se hace referencia al estudio de sistemas vivos, sociales y psíquicos que son intencionados; capaces de pensamiento racional; de conciencia de sí y de su entorno; y de llevar a cabo procesos de reflexión acerca de su actividad y de las relaciones que establecen en los distintos niveles del sistema de enseñanza y aprendizaje. En ese contexto, los artefactos mediadores son parte de un proceso de enseñanza y aprendizaje global y de coordinación de variables, con especial énfasis en los elementos cibernéticos.

El modelo general de un sistema de enseñanza y aprendizaje propuesto por Engeström es de especial interés ya que, como se mencionó, integra los postulados de la teoría de sistemas, la teoría de la complejidad y la teoría de la actividad, y propone la enseñanza como un sistema de actividad humana. En la figura 5 se hace una descripción general del modelo, donde el triángulo interno vincula al personal docente con el objeto de su actividad: el aprendizaje y la formación del estudiantado, y las relaciones con la comunidad académica, lo que evidencia que la práctica docente -la acción del profesorado y el pensamiento que le subyace- no es una actividad estrictamente individual o colectiva, sino una cuestión que envuelve aspectos personales, educativos, socioculturales e institucionales. Estricto sensu, el sistema de enseñanza se despliega en un sistema didáctico, cuyos componentes son: el profesorado, el estudiantado, el saber enseñado y las relaciones entre ellos (Chevallard, 2009).

El triángulo externo de la figura 5 indicaría que sobre la práctica docente influyen otros factores o conjuntos de acciones y experiencias concretas que, eventualmente, se convierten en obstáculos, limitaciones, oportunidades o posibilidades para su desarrollo: las reglas y convenciones de la institución sobre la enseñanza, la división del trabajo académico y los artefactos mediadores que utiliza y se apropia el personal docente para realizar su trabajo. Los artefactos mediadores pueden ser: espacios, tecnologías de la comunicación y la información y otras; libros y todo tipo de materiales impresos, recursos físicos y discursos.

En cuanto al rol y funciones del profesorado en un sistema de enseñanza y aprendizaje de nivel universitario, el modelo de Engeström permite establecer conceptualmente la existencia de un ámbito específico de acción del profesorado dentro del sistema, con clara diferencia de sus otros componentes. Si bien la práctica docente está atravesada por diferentes determinaciones (triángulos $\mathrm{A}, \mathrm{B}, \mathrm{C}$ y D), también hay un grado de autonomía relativa en las acciones del personal académico, que tiene que ver con sus características personales, profesionales, laborales y docentes. En ese nivel emerge el rol y función específica del profesorado: la enseñanza; y, con ello, la actividad docente en su especificidad. 


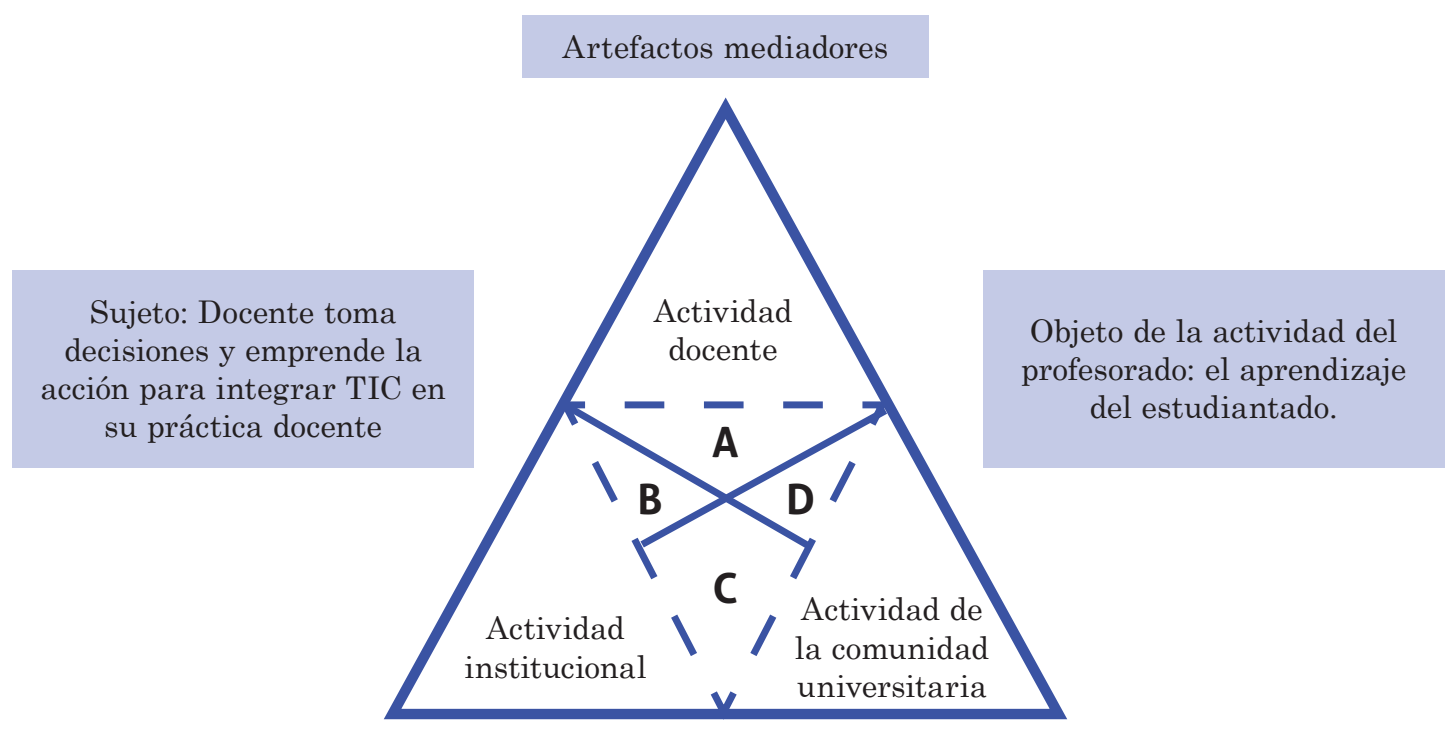

Reglas y convenciones en relación con la evaluación, la estructura de las asignaturas, la forma que debe adoptar la enseñanza, los niveles, los requisitos previos de aprendizaje, etc.
Comunidad: las comunidades de docentes, la comunidad de docentes y estudiantes, las comunidades de estudiantes.
División del trabajo: ¿Qué hace el profesorado? ¿Qué hace cada estudiante? ¿Qué hace el estudiantado como grupo?

Figura 5. Elementos de un sistema de enseñanza y aprendizaje, según el modelo de Engenström. Construcción propia con base en Knight (2006).

Si se entiende la enseñanza como "una selección de actividades diseñadas para provocar un complejo aprendizaje del estudiante" (Knight, 2006, p. 128), ello incluye: "la docencia cara a cara y otras modalidades, las tareas para el aprendizaje, la información sobre las consecuencias y el diseño de las asignaturas, los programas y los ambientes de aprendizaje; [es decir, la enseñanza es] la creación deliberada de situaciones a partir de las cuales unos aprendices motivados no deben poder salir sin aprender o formarse"(Knight, 2006, p. 128).

La docencia o enseñanza como principal función del profesorado remite al aprendizaje. De las actuaciones del personal docente depende, en alto grado, el aprovechamiento de sus estudiantes, porque la enseñanza tiene como fin que ocurra el "aprendizaje" como resultado de la intervención y guía del profesorado; no obstante, el aprendizaje que acontece en las instituciones educativas no es cualquier aprendizaje, sino que está "deliberadamente definido" en el nivel formal, pedagógico-curricular y práctico. En el nivel formal, por medio de las reglas y disposiciones de la institución educativa; en el pedagógico-curricular, a través de la filosofía y teoría educativa elegida, que se concreta en los planes de estudio y programas de los cursos, y a partir de las actuaciones del profesorado, que se orienta por sus conocimientos sobre el campo disciplinar y la enseñanza. Esto coloca al personal docente en un lugar privilegiado dentro de un sistema de enseñanza y aprendizaje, que vincula el éxito del aprendizaje del estudiantado con la formación profesional y docente del personal académico y, por ende, con el 
logro de las metas de la institución educativa como un todo. Este hallazgo es fundamental, ya que indica que el saber disciplinar, por amplio y profundo que sea, resulta insuficiente para una adecuada práctica de la enseñanza en educación superior. Las generaciones actuales de docentes de nivel universitario marcan un punto de inflexión entre la docencia intuitiva y la docencia profesional, en tanto saber diferenciado del saber disciplinar.

En la figura 6, se presentan los principales componentes de una institución universitaria, donde la docencia ocupa el lugar central, porque circunscribe el propósito general de la educación superior. La función de la universidad es la formación profesional, por lo que la docencia constituye el punto en el cual convergen los demás componentes de un sistema de educación superior. De ahí, la importancia y el carácter que adquiere el personal académico, en tanto actor imprescindible y clave en el proceso de enseñanza y la gestión del aprendizaje.

Políticas de educación superior

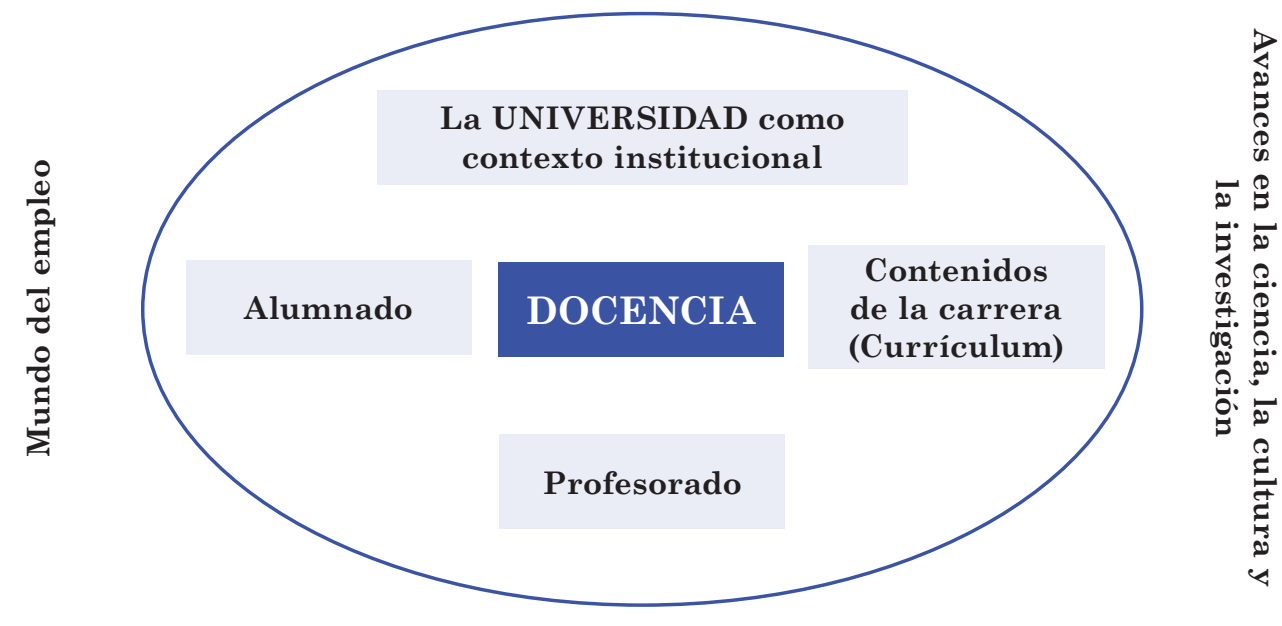

Colegios profesionales y sistemas de acreditación

Figura 6. Componentes principales de las instituciones de educación superior (Zabalza, 2002, p. 12).

Si bien en todo sistema de enseñanza y aprendizaje las competencias de los distintos actores y actoras están "delimitadas con precisión, los registros están asignados, las responsabilidades distribuidas y los poderes circunscriptos" (Chevallard, 2009, p. 29), el profesorado es el principal recurso para la docencia universitaria y lo conforman individuos con características personales, culturales y profesionales particulares, que inciden en la forma como se construye el conocimiento y se le presenta al estudiantado. El personal docente también influye de forma decisiva en la manera de concebir el trabajo académico y la formación profesional. Por esa razón, ninguna innovación o cambio educativo son posibles al margen de 
quienes llevan a cabo la docencia; por el contario, "los profesores se convierten siempre en los mediadores y agentes básicos de las innovaciones en la Universidad (siempre como aplicadores y, con frecuencia, como instigadores y planificadores de los cambios)" (Zabalza, 2002, p. 14).

La fundamentación teórica de que el personal docente desempeña un papel esencial en la estructura y funciones de toda institución educativa, incluidas las universidades, requiere de un estudio contextual del profesorado que visibilice los elementos que intervienen en su práctica y la afectan para desarrollarla o limitarla. Esa exigencia se incrementa cuando se está en una fase de adaptación de la educación superior a una nueva realidad histórico-social, donde las demandas educativas han cambiado de manera radical. En la actualidad, la "universidad moderna está lejos de ser la 'ideal': una comunidad académica estable, libre de la interferencia externa y dedicada a la persecución, preservación y diseminación del conocimiento disciplinario" (Monereo y Pozo, 2003, p. 119). Para adecuar la universidad a los requerimientos de formación profesional de la sociedad contemporánea se deben revisar "sus funciones, sus formas de organización, el sentido de la docencia y el protagonismo del profesor, entre otras cuestiones" (Monereo y Pozo, 2003, p. 119).

En la figura 7, se esquematiza una primera contextualización del fenómeno de la docencia, para situar la actividad del profesorado en un escenario de transformación de la universidad como un todo, en particular, respecto de su significado y el protagonismo del personal académico. Ello involucra las políticas de la universidad acerca del personal académico, el perfil institucional del profesorado, el currículo, el modelo de enseñanza y las acciones institucionales orientadas a favorecer la profesionalización de la docencia, el reciclaje profesional y la formación continuada del personal académico.

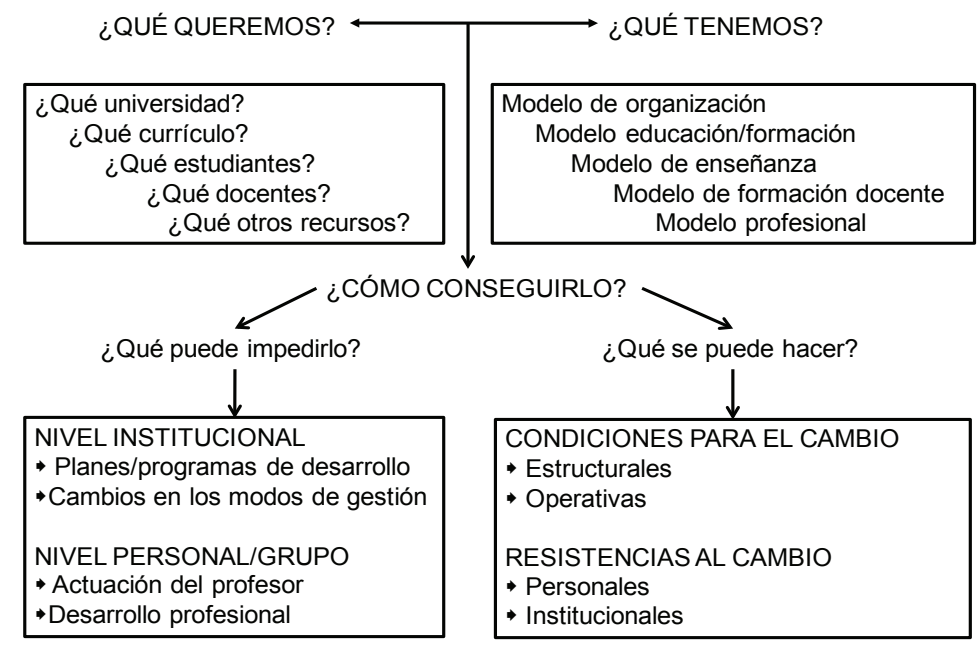

Figura 7. Elementos para un estudio contextual del profesorado universitario (Monereo y Pozo, 2003, p. 120).

El modelo de Engeström muestra que entre los factores que inciden en el cambio en educación superior hay aspectos institucionales, disciplinares y personales. En el nivel institucional, se distinguen los estructurales y operativos. En el disciplinar, el avance del 
conocimiento y en el personal, la resistencia al cambio que se puede manifestar tanto en grupos como en personas. La docencia es uno de los factores que "más sustancialmente condicionan el desarrollo de la Universidad y de la formación que se planifica y desarrolla en su seno" (Zabalza, 2002, p. 15).

El cambio en las universidades ha tenido un impacto profundo en las exigencias y características del trabajo docente, principalmente, en cuanto a la calidad de la enseñanza, y los enfoques y estrategias de actuación del profesorado. Las nuevas demandas a este sector tienen repercusiones en diversos ámbitos: ampliación de las funciones tradicionales, exigencia de mayores esfuerzos en la planificación, diseño y elaboración de las propuestas docentes e incremento de la burocratización didáctica (Zabalza, 2002).

Las exigencias emergentes al personal académico evidencian que hay una complejización creciente de la docencia universitaria, que requiere una reconstrucción y resignificación de su perfil y funciones. Si bien hay un debate abierto al respecto, resulta indiscutible que las lecciones magistrales y la explicación tradicional de contenidos son insuficientes en el nuevo escenario de la educación superior. La actuación docente ocurre hoy en un contexto de transformaciones profundas de las universidades, que conlleva retos para el sector en diferentes niveles. Uno de ellos, ampliamente reconocido, es la incorporación de las tecnologías de información y comunicación (TIC) en la enseñanza y la creación de formas innovadoras de docencia que aprovechen el potencial de esos medios para elevar la calidad, equidad, acceso y pertinencia de la formación universitaria. Ello obedece a problemas y exigencias educativas nacionales y globales, que implican un abordaje sistémico y un enfoque que permita una perspectiva realista de las implicaciones que esto tiene para la docencia universitaria.

Siguiendo el modelo de Engeström, las condiciones que se requieren para el cambio en la docencia universitaria involucran el sistema de enseñanza y aprendizaje como un todo. Esto supone transformaciones estructurales, funcionales, pedagógico-curriculares, culturales, operativas y en la enseñanza misma, que dependerán de la gestión de la innovación, de la forma en que se maneje la resistencia al cambio, así como de la capacitación, el apoyo institucional y las posibilidades reales de las personas clave involucradas en el logro de esa meta; en particular, del profesorado. Desde esta perspectiva, el modelo de un sistema de enseñanza y aprendizaje de Engeström tiene como principal ventaja que rompe con perspectivas teóricas parciales y parcializadas, así como con posiciones idealistas, dogmáticas, ideológicas, politizadas y reduccionistas de la docencia.

Las tendencias actuales en el sector universitario, consecuencia de las transformaciones del mundo contemporáneo, quedaron selladas en la Conferencia Mundial sobre la Educación Superior: Visión y Acción (UNESCO, 1998a), específicamente, en cuanto a la necesidad de "contribuir al desarrollo y la mejora de la Educación en todos los niveles, en particular mediante la capacitación del personal docente" (UNESCO, 1998b). Los principales cambios en la enseñanza a escala mundial se vinculan con los aportes de nuevas teorías y enfoques sobre la enseñanza y el aprendizaje, y sus implicaciones en la conceptualización de la enseñanza, el currículo, las tareas y actividades que realizan docentes y estudiantes; la mediación social, los artefactos mediadores y la evaluación, como se resume en la tabla 1. 
Tabla 1

Cambios en aspectos esenciales de la enseñanza, el aprendizaje y la educación

\begin{tabular}{cll}
\hline \multicolumn{1}{c}{ Aspecto } & \multicolumn{1}{c}{ Ahora } & \multicolumn{1}{c}{ Antes } \\
\hline \multirow{2}{*}{ Aprendizaje } & Construcción activa & Dar información \\
& Conexiones & Jerárquico \\
& Situado & Descontextualizado \\
\hline \multirow{2}{*}{ Enseñanza } & Transformación & Transmisión \\
& Andamiaje & Directo \\
\hline Currículo & Maleable & Fijo \\
\hline \multirow{2}{*}{ Tareas } & Auténticas & Aisladas \\
& Conjunto de representaciones & Materiales secuenciados \\
\hline \multirow{2}{*}{ Mediación social } & Comunidad de aprendices & Individual \\
& Colaboración & Competición \\
& Discurso & Recitación \\
\hline \multirow{2}{*}{ Herramientas } & Uso integrado e interactivo de & Papel y lápiz \\
& los ordenadores & Pruebas de rendimiento \\
& Basado en la actuación & Test estandarizados \\
\hline \multirow{2}{*}{ Evaluación } & Carpetas individuales &
\end{tabular}

Nota: (Monereo y Pozo, 2003, p. 122).

Las transformaciones en las demandas educativas de la SIC obligan a las instituciones universitarias a transformarse de manera radical en el corto plazo. Ello incluye la organización y culturas académicas y el perfil y características del profesorado, pues su función es el ejercicio de la docencia. Que el personal académico modifique su práctica docente en forma integral y asuma un rol completamente otro en el proceso de enseñanza requiere, además de motivación e interés personal, de apoyo institucional y oportunidades de formación profesional y docente que le faculten para ello a lo largo de su vida laboral.

El nuevo profesorado debe dejar prácticas propias de la enseñanza tradicionaltransmisionista y conductista e implementar otras donde se desempeñe como orientador de los aprendizajes. Debe pasar de un entorno laboral donde la evaluación docente es escasa y limitada, a otro donde se la utiliza como una herramienta para la mejora continua. Dejará de trabajar de forma aislada y clausurada para formar parte de entornos de colaboración y colegialidad; pasará de pertenecer a comunidades académicas cerradas sobre sí mismas y para el estudiantado, a conformar nuevas relaciones con colegas y estudiantes, especialmente, como colaborador, asesor y tutor.

$\mathrm{Si}$ los cambios que requieren las universidades para ajustarse a las demandas educativas actuales establecen como punto de partida al profesorado, debido a que las transformaciones fundamentales tienen que ver con la docencia, el personal académico debe caracterizarse por poseer: 
- "Conocimiento científico especializado relacionado con las temáticas que abordan.

- Conocimiento cultural en relación con la materia de enseñanza y el mundo general de la cultura.

- Conocimientos psicopedagógicos, relacionados con teoría del aprendizaje, conocimientos curriculares (programación, evaluación...) y de organización institucional.

- Conocimientos de la práctica docente, relacionados con la experiencia docente, socialización profesional, habilidades comunicativas, recursos metodológicos e instrumentos de reflexión sobre la práctica. Este conocimiento se refiere al que se tiene de las diversas y complejas situaciones que se producen durante la práctica profesional y se relaciona con los dilemas prácticos a los que debe enfrentarse y solucionar un profesional. Las condiciones de inestabilidad, conflicto, singularidad e incertidumbre que acompañan su producción no son reproducibles en el aula, donde impera la falsa certeza de racionalidad técnica.

- Conocimientos sobre sí mismo." (Monereo y Pozo, 2003, p. 128)

Los conocimientos enumerados conllevan ciertas capacidades adicionales, ya que el profesorado debe poseer, además de un amplio bagaje disciplinar, conocimiento en teoría de la educación y didáctica de la disciplina que enseña. De igual forma, debe ser capaz de adaptar "y hacer comprensible el contenido disciplinar a determinado tipo de estudiantes" (Monereo y Pozo, 2003, p. 128). No es lo mismo impartir un curso introductorio que uno avanzado, ni trabajar con estudiantes con experiencia laboral o sin ella. En ese contexto, el profesorado, adicionalmente, debe:

- Conocer los temas enseñados en una determinada asignatura.

- Conocer los aspectos de la materia más difíciles de aprender para los estudiantes y los factores que inciden en su nivel de dificultad.

- Conocer las preconcepciones que los estudiantes tienen sobre determinados temas de estudio.

- Conocer los materiales y recursos utilizables en la materia que se va a enseñar.

- Conocer las formas más útiles de representar ideas: las ilustraciones y explicaciones más poderosas, las manipulaciones y demostraciones más adecuadas, las mejores analogías y ejemplos.

- Conocer las estrategias de enseñanza que hacen que el contenido sea comprensible e interesante para los estudiantes, que facilita el desarrollo conceptual del contenido. (Monereo y Pozo, 2003, pp. 128-129)

Las tareas emergentes del profesorado universitario requieren de nuevas competencias, en especial, en el campo pedagógico, curricular y didáctico. El saber sobre la enseñanza no puede fundarse en intuiciones ni en creencias subjetivas; tampoco puede sostenerse sobre una práctica orientada por el "ensayo-error", a partir de nociones imprecisas de "deber ser" del hecho educativo ni debe continuar como una actividad que se desarrolla en aislamiento, 
clausura y secreto (Salas, 2013). La enseñanza debe tener bases teórico-conceptuales sólidas que orienten la práctica docente en organizaciones educativas donde haya espacios para el debate, la discusión y el análisis de la enseñanza. De ahí, la importancia de la formación pedagógica inicial y continuada del profesorado universitario.

La sistematización, conceptualización y reflexión sobre la práctica docente juegan un papel fundamental en el desarrollo del profesorado, lo que implica un compromiso institucional con la docencia en varios niveles:

a. Medidas estructurales: itinerarios formativos, convocatorias sobre docencia e innovación, reuniones científicas, difusión de experiencias, creación de materiales sobre formato o contenido de la docencia, desarrollo de centros específicos (acreditación, documentación), evaluación de la docencia, incentivación de la buena docencia, reconocimiento institucional de la innovación docente, intercambio de profesores, apoyo continuado al profesorado en las clases, etc.

b. Medidas didáctico-organizativas: reformulación de programas, ajuste de docentes a sus posibilidades como enseñantes, potenciación de departamentos y equipos, organización de tutorías, clarificación de competencias docentes entre facultades, departamentos y titulaciones, asesoramientos específicos, etc.

c. Medidas diferenciales, dirigidas al profesorado novel, al implicado en prácticas, al profesorado de primero, al profesorado experimentado, etc. (Monereo y Pozo, 2003, p. 136)

En procesos de innovación de la docencia, el compromiso de la institución y la alineación de las unidades académicas con las políticas y acciones institucionales son claves para el éxito. La investigación ha mostrado que hay una estrecha relación entre la organización y la estructura académica, la innovación, la evaluación y la formación del profesorado (Monereo y Pozo, 2003).

Un primer paso en las universidades hacia el cambio de la docencia es la configuración de un perfil académico y profesional del profesorado que incluya: investigación, acción social, integración del conocimiento, vinculación con la comunidad académica interna y externa, y docencia. A partir del perfil del personal académico, la administración universitaria estará en mejores condiciones para cimentar las bases para emprender acciones tendientes a favorecerlo y consolidarlo en la institución. Como ejemplo de lo anterior, en la Universidad de Costa Rica se determinó que el profesorado de la institución debe tener las siguientes características para el adecuado ejercicio de la docencia:

- Conocimiento experto en su disciplina.

- Conocimiento de diversas perspectivas pedagógicas, metodologías y técnicas didácticas.

- Capacidad para orientar el proceso de enseñanza-aprendizaje de acuerdo con necesidades de aprendizaje del estudiantado.

- Capacidad para planificar y organizar eficazmente el proceso de la enseñanzaaprendizaje.

- Habilidad para comunicar en forma clara y precisa las ideas y reflexiones. 
- Capacidad para utilizar las diversas tecnologías de la comunicación e información.

- Capacidad de trabajo en equipo con sus pares y el estudiantado.

- Enseñar a aprender al estudiantado.

- Evaluar y retroalimentar el proceso formativo del estudiantado.

- Capacidad para participar activamente en el diseño y mejoramiento del currículum. (Universidad de Costa Rica, 2004)

Debido a la necesidad de favorecer el cambio y la innovación, las organizaciones contemporáneas ensayan nuevas formas de capacitación de sus recursos humanos a lo largo de la vida laboral, para ajustar los perfiles socio-profesionales y labores a las circunstancias y el contexto. Las instituciones educativas no son la excepción y, entre ellas, destacan las universidades, ya que el personal académico, generalmente, no es contratado para el ejercicio del cargo con conocimientos previos y especializados en docencia universitaria. Hasta ahora, en Costa Rica y muchos países del mundo, el único requisito para ejercer la docencia en educación superior es el grado académico en el campo disciplinar. En vista de ello, muchos profesores y profesoras asumen su práctica docente de manera intuitiva y por modelaje de sus experiencias como estudiantes a lo largo de su formación en secundaria y la universidad (Salas, 2013). Pocos docentes universitarios estudian la enseñanza en educación superior por su cuenta y los que asumen el riesgo, por lo general, enfatizan en áreas de su interés, sin un corpus teórico-conceptual articulado y coherente que permita una práctica pedagógica ajustada a estándares deseables, de acuerdo con el grado de conocimiento científico alcanzado en esa materia y las necesidades educativas emergentes.

\section{Conclusiones}

Las carencias teórico-conceptuales que caracterizaron la educación como campo de estudio en la Era Industrial y a lo largo del siglo XX (Luhmann, 1996), se han subsanado gracias a los avances de las últimas tres décadas en las ciencias sociales y al desarrollo científico en la disciplina, que cuenta en la actualidad con un corpus de teorías diverso, consistente y articulado en distintos paradigmas, que van desde la postura epistemológica empírico-analítica hasta la sistémico-compleja. Estos ofrecen un marco de referencia vasto, enriquecido constantemente por los avances científicos y los hallazgos de investigación en todas las disciplinas que nutren de una forma u otra la comprensión del fenómeno educativo y el desarrollo de tecnologías necesarias para atender los problemas que le son propios.

Ante el desarrollo científico alcanzado en educación, no se justifica la persistencia de la atención empírica, subjetiva e intuitiva de las situaciones educativas por parte del profesorado. El desafío de nuestro tiempo es reconocer que el desarrollo del conocimiento en educación superó la fase pre-científica y que en la actualidad constituye un campo disciplinar que cumple con todos los requerimientos establecidos para adquirir el estatus de ciencia (Bunge, 1992), recuperando la enseñanza del nivel de "arte" al de "actividad humana particular", inscrita en un sistema de enseñanza y aprendizaje socio históricamente determinada. 
La trayectoria de una fase pre-científica a una científica ha ocurrido en todas las disciplinas, de acuerdo con las evidencias de la historia de la ciencia, que documenta procesos de transición de un estado a otro, cuya característica común es ser problemáticos, porque muchos intereses de personas y de las mismas comunidades científicas, así como de índole económica, sociocultural e ideológica inciden en su avance y desarrollo (Kuhn, 1980). A ello, se suman la resistencia al cambio y la innovación individual y colectiva, las fuerzas del anquilosamiento del statu quo, y la entronización de zonas de confort e intereses de grupos, cuya detección y esclarecimiento no son fáciles.

El modelo de un sistema de enseñanza y aprendizaje desarrollado por Engeström tiene un enorme potencial teórico-conceptual y práctico para la descripción, explicación y comprensión del fenómeno educativo en general y de la docencia en particular, desde una perspectiva sistémica capaz de articular la complejidad que le subyace. Situaciones donde entran en juego tantos factores y variables como es el caso de la docencia, debido a las intrincadas fuerzas y vicisitudes que la atraviesan en los niveles personal, grupal, sociocultural, económico, político, institucional y contextual, son extraídas finalmente de "la caja negra" que por décadas favoreció y legitimó prácticas de enseñanza en educación superior fundamentadas en razones ajenas a la "racionalidad científica". En la actualidad, no hay justificación para proceder de esa forma; tampoco el contexto de la SIC y las demandas educativas emergentes lo permiten.

La falta de formación pedagógica del personal académico constituye, en la actualidad, uno de los principales problemas a solventar en la gestión del cambio y la innovación en la docencia universitaria. Ante esta realidad, el modelo de Engeström es una oportunidad para hacer investigación científica que oriente la toma de decisiones en ese ámbito y realizar los cambios que correspondan con base en datos e información, para poner la docencia universitaria a la altura de los tiempos.

De acuerdo con lo expuesto, el modelo de Engeström es un marco de referencia teóricoconceptual y epistemológico robusto que favorece la investigación científica situada y contextualizada de la docencia en sus dimensiones fundamentales, que ofrece respuesta a las preguntas tradicionales de la educación en esa materia, así como a muchas otras que han constituido misterios o enigmas que sustentaron la tesis de que la "enseñanza es un arte". El principal aporte del modelo es, entonces, el reconocimiento y explicitación de las determinaciones sociohistóricas, culturales e institucionales que subyacen a la docencia, porque permite superar la perspectiva mágica, deshistorizada e idealizada que la ha marcado, ya que revela que el profesorado no tiene autonomía ni control absoluto sobre su actividad como "enseñante", sino que se encuentra inmerso en contextos institucionales, disciplinares, socioculturales y pedagógicos que modelan las características y calidad de la práctica de la enseñanza.

Como ocurre en el ámbito científico, toda aportación epistemológica y teórico-conceptual para la mejor comprensión de los fenómenos permite el conocimiento necesario para encontrar soluciones a los problemas y desafíos que conllevan; por ello, nos permitimos afirmar que el modelo de Engeström es una oportunidad clave para investigar y producir conocimiento nuevo sobre la docencia universitaria, en un escenario global donde la universidad como institución está inmersa en fuertes presiones de diversa naturaleza, tanto nacionales como internacionales, para que cambie y responda a las demandas educativas del presente. 


\section{Referencias}

Bunge, M. (1992). La investigación científica. México: Ariel.

Chevallard, Y. (2009). La transposición didáctica. Del saber sabio al saber enseñado. Buenos Aires: Aique.

Delgado, J. M. y Gutiérrez, J. (1998). Métodos y técnicas cualitativas de investigación en ciencias sociales. Madrid: Síntesis.

Flórez, R. (2000). Docente del siglo XXI. Cómo desarrollar una práctica docente competitiva. Colombia: McGraw-Hill.

Frade, L. (2009). Desarrollo de competencias en educación: Desde preescolar hasta el bachillerato. México: Inteligencia Educativa.

Freud, S. (1981). Obras completas. Madrid: Biblioteca Nueva.

García, J. y García, A. (1996). Teoría de la educación I. Educación y acción pedagógica. Salamanca: Universidad.

Habermas, J. (1988). Teoría de la acción comunicativa. Crítica de la razón funcionalista. Tomo II. España: Taurus.

Hargreaves, A. (2003). Profesorado, cultura y posmodernidad. Cambian los tiempos, cambia el profesorado. Madrid: Morata.

Kaplún, M. (1998). Una pedagogía de la comunicación. Ediciones de la Torre, Madrid, España.

Knight, P. (2006). El profesorado de educación superior. Formación para la excelencia (2 ${ }^{\mathrm{da}}$. Ed.). Madrid: Narcea.

Kozma, R. (Ed.). (2003). Technology, innovation, and educational change. A global perspective E.U.A.: ISTE.

Kuhn, T. (1980). La estructura de las revoluciones científicas. Madrid: Fondo de cultura económica.

Luhmann, N. (1996). Teoría de la sociedad y pedagogía. España: Paidós.

Luhmann, N. (1998). Sistemas sociales. Barcelona: Anthropos.

Monereo, C. y Pozo, J. (2003). La universidad ante la nueva cultura educativa. Madrid: Síntesis. 
Padrón, José. (2007). Tendencias epistemológicas de la investigación científica en el siglo XXI. Cinta de Moebio, 28, 1-28.

Salas, F. (2013). La integración de las TIC en la docencia en la UCR: Estudio desde un enfoque sistémico-complejo del personal docente que se certificó en el curso Educar para el futuro, versión universitaria en el período 2003-2006 (Tesis doctoral). Universidad de Costa Rica, San José, Costa Rica.

Vidal, G. (2007). La actividad del profesor. Recuperado de http://www.educar.org/articulos/ LaActividaddelProfesor.asp

Vygotski, L. S. (1995). Obras escogidas (Tomo III). Madrid: Visor.

Vygotski, L. S. (2001). Psicología pedagógica. Argentina: Aique.

Vizcarro, C. y León, J. A. (1998). Nuevas tecnologías para el aprendizaje. Madrid: Pirámide.

Zabalza, M. (2002). La enseñanza universitaria. El escenario y sus protagonistas. Madrid: Narcea.

Zabalza, M. (2003). Competencias docentes del profesorado universitario. Calidad y desarrollo profesional. Madrid: Narcea.

UNESCO. (1998a). La educación superior en el siglo XXI: Visión y acción. París: UNESCO.

UNESCO. (1998b). La formación del personal de la educación superior: Una misión permanente. París: UNESCO.

Universidad de Costa Rica. (2004). La Gaceta Universitaria No. 39. Consejo Universitario, sesión 4932. Recuperado de http://www.cu.ucr.ac.cr/uploads/tx ucruniversitycouncildatabases/ officialgazette/2004/g39-2004.pdf 